\title{
Philosophiques
}

\section{Écrire la société : d'une dérive à la limite du réel et du fictif}

\section{Nicole Brossard}

Volume 21, numéro 2, automne 1994

Les femmes et la société nouvelle

URI : https://id.erudit.org/iderudit/027274ar

DOI : https://doi.org/10.7202/027274ar

Aller au sommaire du numéro

Éditeur(s)

Société de philosophie du Québec

ISSN

0316-2923 (imprimé)

1492-1391 (numérique)

Découvrir la revue

Citer ce document

Brossard, N. (1994). Écrire la société : d'une dérive à la limite du réel et du fictif. Philosophiques, 21(2), 303-320. https://doi.org/10.7202/027274ar

\section{Résumé de l'article}

Que la situation de certaines femmes se soit améliorée, cela ne signifie pas que la condition des femmes en général a changé en profondeur, et que le féminisme peut prendre congé. Au contraire, à la fois comme pensée, comme discours, comme pratique et comme éthique, le féminisme reste indispensable à l'avènement d'un monde meilleur. d'utilisation que vous pouvez consulter en ligne.

https://apropos.erudit.org/fr/usagers/politique-dutilisation/ 


\title{
CONFÉREN CE INAUUURALE
}

\section{Écrire la société : d'une dérive à la limite du réel et du fictif}

\author{
par \\ Nicole Brossard
}

\begin{abstract}
RESUME : Que la situation de certaines femmes se soit améliorée, cela ne signifie pas que la condition des femmes en général a changé en profondeur, et que le féminisme peut prendre congé. Au contraire, à la fois comme pensée, comme discours, comme pratique et comme éthique, le féminisme reste indispensable à l'avènement d'un monde meilleur.
\end{abstract}

ABSTRACT : Although some women fare better in our society, this does not mean that the situation of women in general is no longer problematic, and that feminism is no longer needed. On the contrary, as a way of thinking, as a discourse, as a praxis and as an ethics, feminism remains essential to the coming of a better society.

Si ce n'était du désir, de quelques utopies et des visages de femmes que je porte en moi, je crois que je pourrais tenir un discours raisonnable. Je pourrais faire le tour de la question féministe sans jamais entrer au cœur de ce qui la motive. Je pourrais garder la bonne distance sociologique et rendre compte, sans trop d'émotion, des changements engendrés par le féminisme et des résistances qu'il rencontre. Mais l'émotion, j'y tiens car c'est elle qui a nourri ma réflexion et ma motivation chaque fois quej'ai eu l'impression de comprendre un peu la vaste imposture de la pensée patriarcale, c'est elle qui m'a permis de dépasser les bornes et de m'aventurer toujours un peu plus loin dans ce qu'on appelle la réalité des idées qui gravitent autour de l'étrange monde du rêve et de la fiction. Et ce monde-là, qu'on le veuille ou non, est un monde précieux pour qui nourrit quelque espoir sur le futur de l'espèce. L'émotion,j'y tiens parce qu'elle est une preuve du vivant, parce qu'elle m'oblige à m'inquiéter de la réalité, à imaginer en deçà et au-delà des images familières, à dériver dans des zones inconnues. Ce que je veux, c'est comprendre ce qui, au fond de la conscience, tremble comme une énergie qui déplace constamment 
le désir, le savoir et l'angoisse. Je veux penser avec des reflets dans les yeux, comprendre comment ce qui se décide dans la fiction et dans l'imaginaire construit notre rapport à la réalité. Je pense que la réalité ne dépasse pas la fiction. Il me semble plutôt que l'une et l'autre se nourrissent mutuellement de ces possibilités que sont les métaphores et les narrations engendrées dans le chassé-croisé des sensations, des émotions et du savoir. L'émotion, j'y tiens parce qu'elle exacerbe en moi un état d'esprit sans lequel je me contenterais d'un peu d'égalité et d'équité.

Aussi, je doute que quelques lois (favorisant l'extension des droits et du rôle des femmes dans la société) aient pu, en deux décennies et demie, modifier considérablement les méthodes d'intimidation développées à travers les mœurs et les institutions patriarcales. Ces méthodes demeurent les mêmes : logiques du deux poids, deux mesures, jeux de cartes truquées, siphonnage d'énergie, mépris, viols et chantage.

Quoi qu'on en pense, l'autonomie et l'épanouissement des femmes demeurent soumises à la fluctuation des mœurs patriarcales. Tantôt libérale, la société décriminalise ou tolère la prostitution, la pornographie, l'usage des drogues, l'homosexualité, les unions libres, et relâche son contrôle sur le ventre des femmes. Tantôt conservatrice, la société renforce la surveillance des femmes en les réinsérant, sécurité maximum, dans le cadre du mariage, interdit l'avortement, laisse à Dieu, à la Nature ou à la Patrie de décider si la pornographie est salubre activité et si la prostitution est vraiment le plus vieux métier du monde. Dans une société conservatrice, le féminisme est un outil de survie; dans une société libérale, le féminisme est un espace de conscientisation qui permet aux femmes de se reconnaître comme groupe et de s'épanouir comme individus.

La domination d'un groupe par un autre passe par les institutions et/ou par les individus. Si dans une société conservatrice la loi des hommes se charge de garder les femmes en position d'infériorité et de dépendance, dans une société libérale comme la nôtre, les hommes ont plus ou moins individuellement à charge soit de maintenir leurs privilèges, soit de s'assurer la collaboration des femmes en entamant avec celles-ci de nouveaux rapports. Dans le premier cas, les hommes redoubleront d'ardeur et d'astuces sinon de violence pour garder les femmes à leur place, c'est-à-dire sous leur contrôle; dans le deuxième cas, les hommes accepteront de développer des attitudes et des comportements nouveaux.

C'est exactement ce qui se passe dans notre société mais on oublie trop facilement que la majorité des hommes penchent pour la première solution et que d'une manière générale la misogynie continue d'écouler son stock de propagande à travers le cinéma, la vidéo-clip, la pornographie, la publicité. Ce que l'on donne d'une main (loi contre le harcèlement sexuel, manuels scolaires non sexistes), on le reprend de l'autre sans ménagement.

Aussi les femmes sont-elles encore représentées comme des objets décoratifs, sont-elles encore perçues comme des entités négligeables dont le 
sort dépend de la bonne volonté des hommes politiques et de la santé mentale des hommes de la rue et du foyer. Les femmes demeurent des majoritaires minorisées et marginalisées dans le grand ensemble humain. La liberté accordée aux femmes est une liberté surveillée.

Ce n'est pas parce que la société contemporaine a permis à quelques femmes d'agrandir leur champ de compétence, d'entrer dans le jeu de la compétition et d'augmenter leur pouvoir de dépenser que la condition des femmes s'en trouve changée au point qu'il faille signifier son renvoi au fẻminisme.

Pourtant, il semble y avoir dans l'air un consensus qui tend à conclure que les droits obtenus par les femmes et les concessions consenties aux féministes témoignent d'une victoire du féminisme, consensus qui a le double effet de rendre excessives et conséquemment irrecevables d'éventuelles revendications féministes mais aussi qui permet de clore le débat en affirmant la caducité du discours féministe. Ainsi parlera-t-on de postféminisme pour désigner une relative autonomie des femmes à l'intérieur d'un consensus social renouvelé où les hommes et la loi offriraient aux femmes des garanties de plein épanouissement.

Sur le plan politique, le postféminisme pourrait alors être considéré comme un droit de regard et de paroles acquis dont l'usage serait délégué à quelques fonctionnaires et lobbyistes qui veilleraient ainsi aux intérêts des femmes. Dans la vie privée, le postféminisme se traduirait par la capacité, nouvellement acquise, de chaque femme à organiser et à gérer son bonheur ainsi qu'à faire respecter ses droits par son entourage immédiat. Ainsi une femme dans la quarantaine me disait récemment : «Je vis comme une féministe mais je ne suis pas féministe ». Désinvolte manière de dire « je profite personnellement des acquis du féminisme mais ne comptez pas sur moi pour ce qui est de la solidarité, en action ou en réflexion ».

Or, ce que cette femme et bien d'autres oublient, c'est que chaque fois que la pensée féministe et le discours féministe battent en retraite de la vie sociale et culturelle, c'est tout un environnement propice au développement des femmes (comme individu) qui se dégrade, c'est le statut des femmes comme groupe qui régresse, laissant par la suite à chaque femme le soin de s'expliquer seule au milieu des rires, des quolibets et des insultes.

Le féminisme n'a pas pour seule fonction de voir à ce que justice soit rendue aux femmes. Le féminisme est un environnement propice à la solidarité, à la motivation, à la confiance en soi, à la présence d'esprit, à l'expression et à la création des femmes. Contrairement à ce que l'on aimerait faire croire, le féminisme n'enlève pas aux femmes leur individualité, leur singularité, leur « personnalité ». Bien au contraire, il est un environnement qui permet aux femmes de se développer individuellement. Sans la tension et la dynamique suscitées par le discours féministe dans une société sexiste, sans la solidarité, les célébrations et le sens d'appartenance que stimule le féminisme, la vie normale, c'est-à-dire hétérosexiste, reprend chaque fois son cours comme si de rien n'était. 
Contrairement aux suffragettes et aux féministes libérales qui appuyaient leurs revendications sur les principes d'égalité et de justice, les féministes radicales des années ig7o ont dû découvrir elles-mêmes les principes au nom desquels elles feraient non seulement valoir leurs droits mais aussi leur différence et le sens de leur vie.

Aussi, je ne crois pas qu'on puisse faire un bilan du féminisme des vingtcinq dernières années et imaginer le rôle qu'il aura dans le futur de la société, sans analyser chacun des éléments qui font du féminisme une pensée, un discours, une pratique et une éthique, sans montrer comment la parole en liberté et l'émotion qui ont alimenté le mouvement des femmes se sont, au fil des années, transformées en un corpus de connaissances à l'intérieur duquel on peut, aujourd'hui, réfléchir, imaginer, agir et encore nourrir la passion et le rêve d'un monde meilleur.

Entre la gamme des émotions fortes qui ont contribué à l'expression du point de vue féminin sur les rapports hommes-femmes et les prescriptions qui caractérisent le discours féministe d'aujourd'hui, il y a la gestation d'une pensée.

Je ne voudrais pas ici donner l'impression de vouloir établir un clivage entre subjectivité féminine, pensée féministe et discours féministe mais il me semble que pour mieux témoigner des acquis du féminisme et des résistances qu'il rencontre, il faut s'attarder à comprendre les tâches spécifiques accomplies par l'une et l'autre de ces dimensions du féminisme.

\section{L'expression de la subjectivité des femmes}

On peut penser que depuis toujours les femmes se sont raconté entre elles des histoires de femmes et qu'elles aient, à l'occasion, tenté de s'exprimer, dans le cadre intime de la relation conjugale, sur leurs besoins, leurs frustrations, leurs désirs. Ces paroles de femmes, elles sont à tout jamais perdues dans l'histoire, mais l'histoire nous porte à croire qu'elles ne furent jamais prises au sérieux. Ce n'est que tout récemment que les femmes se sont autorisées à parler publiquement de leur vie privée. Au début, le public était restreint, composé de quelques femmes. C'est ce que l'on a appelé les groupes de prise de conscience. Puis, ces mêmes femmes ont ravalé leurs larmes et s'en sont allées marcher dans les rues en brandissant au bout de leurs bras ces petits résumés de désir et de colère que sont les slogans. Puis, d'autres femmes sont venues témoigner, déclenchant alors une réaction en chaîne de « moi aussi », une avalanche de révélations toutes aussi troublantes les unes que les autres.

L'expression de la subjectivité des femmes sur la place publique est ce qui a permis de nommer le malaise, le tourment, la souffrance, le vertige qui minent les rapports hommes-femmes. Elle a permis de connaître les besoins spécifiques associés au corps des femmes, c'est-à-dire à leur santé physique et psychique et à leur sexualité. Elle a permis de comprendre ce que vivent les femmes en regard de la maternité, de l'amour et de la sexualité. Elle nous a 
aussi fait comprendre qu'amour et sexualité ne riment pas seulement avec hétérosexualité. Elle a aussi provoqué la narration détaillée des actes de violence, de cruauté, d'humiliation dont sont victimes un grand nombre de femmes. Elle a fait le jour sur les inavouables que sont les « histoires » d'inceste et de viol.

En un mot, en exprimant leurs besoins, leurs désirs, leurs frustrations et en racontant leur histoire, les femmes ont littéralement déversé sur la place publique un ensemble d'informations qui, une fois analysées et traitées par le discours féministe, se cristalliseront autour de deux thèmes : la discrimination et la violence systémiques exercées à l'endroit des femmes. Devant la discrimination, on exigera des redressements et des compensations; devant l'ampleur de la violence, on veillera au plus urgent : protéger les femmes par la prévention (information, conseils) et assurer un soutien aux victimes.

C'est autour de ces deux éléments que les féministes justifieront leurs propositions et leurs revendications dans des domaines aussi variés que ceux de l'éducation, du travail, de la psychiatrie, de la médecine, du droit, voire même de la création artistique. Tantôt, on fera valoir alternativement les principes d'égalité et d'équité, tantôt on fera valoir la différence biologique, tantôt la différence résultée du sexisme, tantôt on formulera de nouveaux concepts tel le harcèlement sexuel.

\section{De la lutte contre la discrimination sexiste a a la lutte contre la discrimination}

En décrivant leurs tâches quotidiennes : préparer la nourriture, changer des couches, nettoyer et ranger, en parlant de leurs menstruations, de l'allaitement, de l'accouchement, de leurs « chaleurs », de leur sexualité, les femmes ont fait surgir du corps dans leurs paroles. Beaucoup de corps et de matière. Corps de douleur et corps de plaisir, corps de mal-être et corps de bien-être. Corps : matière à différence à partir de laquelle s'élaborera un discours sur la différence et la normalité. Ainsi, la revendication de la différence, restée longtemps tabou et refoulée comme argument préjudiciable aux demandes fondées sur l'égalité, va-t-elle entraîner tout un discours qui conclura à la nécessité du respect de la différence. Ce discours est un apport capital du féminisme car il aura pour effet d'entraîner et de légitimer une multiplicité de revendications qui seront d'abord fondées sur le respect de la différence, puis sur le droit à la différence. Ainsi verra-t-on s'accroître le nombre des groupes de pression et de revendications qui réclameront un changement d'attitude à leur égard et des mesures concrètes pouvant améliorer leurs conditions de vie, leur permettre ainsi de mieux participer à la société (les handicapé/es), de mieux vivre et exprimer leur préférence sexuelle (gais et lesbiennes), de développer leur identité (les minorités ethniques) ou tout simplement de se prémunir contre les agressions racistes du groupe majoritaire.

Ainsi en cherchant à retrouver leur « humanité » et leur dignité, les femmes contribuent-elles à humaniser les attitudes et les lois à partir desquelles s'ordonnent les rapports humains. Pourtant, par un étrange retour des 
choses, on constate que les législateurs et les citoyens sont de plus en plus enclins à confondre les revendications de la moitié de la population et celles des groupes minoritaires. J'avoue cependant qu'en cela les féministes contribuent elles-mêmes à créer la confusion. Ainsi dans une déclaration récente, la présidente du Comité d'action nationale sur le statut de la femme, affirmaitelle que la clause Canada de l'entente du 7 juillet n'allait pas assez loin dans la reconnaissance de l'égalité des femmes et des minorités. Voulait-elle dire que la représentation des femmes et celle des minorités (souvent représentées par des hommes) devaient être égales?

Faut-il donc rappeler que les femmes ne constituent pas une minorité mais un groupe minorisé qui forme le versant déshumanisé de l'espèce humaine et qu'en cela, nos revendications ne doivent jamais être confondues avec celles des autres groupes, quels que soient les similitudes et les rapprochements que l'on puisse établir entre les groupes dominés et discriminés.

\section{De la dénonciation de la violence faite aux femmes à la dénonciation de la violence}

Ce qui caractérise les actes de violence, c'est qu'ils perdurent toute une vie dans le corps et la psyché des victimes. Une fois étalées au grand jour, les violences corporelles et sexuelles exercées par des hommes à l'égard des femmes, on a eu tôt fait de comprendre que c'était très souvent la petite fille ou l'adolescente en chaque femme qui avait été agressée. Ainsi a-t-on peu à peu levé le voile sur l'inceste, le viol et la brutalité dont sont victimes les enfants. Puis des hommes ont témoigné des violences subies dans leur enfance. A l'occasion, on a pu lire quelques articles affirmant qu'il y avait aussi des hommes battus par leurs femmes. Puis on a porté attention aux mauvais traitements dont sont souvent victimes les personnes âgées. Tout récemment, on pouvait lire dans les journaux que les (hommes) victimes d'agressions physiques et sexuelles alors qu'ils étaient enfants dans les anciennes écoles de réforme d'Alfred et d'Uxbridge en Ontario, recevraient une compensation de i6 millions.

À nouveau, on constatera, qu'en levant le voile sur le secret qui hante leur mémoire d'enfance, les femmes ont fait prendre conscience de la violence souterraine qui mine la réalité conjugale, familiale (là où « normalement » on devrait être en sécurité) et sociale.

Discrimination et violence, ce sont là deux mots clés qui dans une démocratie libérale, engendrent, en principe, blâme et réprobation. Ainsi, on pourrait dire que si, dans un premier temps, les révélations faites par les femmes et politisées par les féministes sont apparues dérangeantes, exagérées et menaçantes, ces mêmes témoignages apparaîtront dans un deuxième temps plus crédibles une fois neutralisés (on parlera alors de victimes, de personnes) ou masculinisés (on parlera d'enfants, d'adolescents, d'orphelins et de vieillards). En fait, ces témoignages seront plus fiables une fois nettoyés de leur spécificité, entendons ici : la discrimination et la violence exercées par le 
groupe hommes sur le groupe femmes. En d'autres termes, ce n'est qu'une fois la violence sexiste transformée en phénomène «social », que la société acceptera d'intervenir.

Il semble donc que les revendications féministes soient un passage obligatoire pour une modification des valeurs dites « humaines », que ces revendications et l'esprit qui les anime forment un pont qui permet à des hommes de reconnaître la détresse d'autres hommes. Aussi, je demeure perplexe devant la question de savoir si les changements que nous initions ne seront pas à long terme réinsérés dans l'universel masculin, reconduisant une fois de plus les questions féminines à leur marginalisation.

On le voit dans le cas de Uxbridge, le système s'est empressé de racheter le « péché » avec beaucoup d'argent, c'est-à-dire de recadrer dans un contexte juridique et monétaire une problématique à caractère moral.

Certes, l'expression de la subjectivité des femmes sur la place publique a permis de faire savoir que l'Autre existe pour de vrai, c'est-à-dire qu'Elle n'est pas insensible à la douleur, au mépris, à l'humiliation, qu'Elle n'est pas un objet (entendre ici inanimée et passive) sur lequel on peut expérimenter à volonté. Ceci, à mon avis, a déplacé une partie de ce qui était antérieurement dans le champ du secret ou de la fiction, dans le champ de la réalité. Par exemple, les scénarios littéraires de viol, d'inceste, de pédérastie jadis élaborés comme des trouvailles érotico-originales exprimant la versatilité créatrice de la libido masculine, ne peuvent plus aujourd'hui être associés au génie et à l'imagination. Malheureusement, la réaction n'a pas tardé à venir. Une partie des fantasmes usés seront réactivés cette fois-ci pour de vrai (je pense ici à la snuff porno). Quant aux nouveaux fantasmes, ils exigeront d'autres scénarios plus osés, plus violents, plus morbides. Je pense, bien sûr, au vidéo-clip mais aussi aux cartes de tueurs en série que les enfants peuvent maintenant trouver dans les paquets de gomme à mâcher qu'ils achètent sur le chemin de l'école. Avouons-le, il y a quelque chose de profondément malade, une aggravation de cette maladie mentale qui consiste à terroriser les femmes et à les humilier. Une femme menacée et terrorisée, on le sait, n'est jamais égale. Ni égale à ellemême, ni égale aux hommes. Il semble que plus les femmes cherchent à comprendre ce qui motive la domination des hommes sur elles, plus elles essayent de trouver des solutions à l'amiable, plus elles tentent d'améliorer, à court terme leur condition, à long terme les relations entre les sexes, plus le système augmente la dose de violence réelle et symbolique.

Certes, l'expression de la subjectivité des femmes aura aussi permis de revaloriser le corps, la mémoire, la subjectivité, thèmes particulièrement développés dans l'écriture au féminin. Dans le cadre d'une quête d'identité, ces éléments agrandissent le champ de connaissance et de vision de chacune, mais en dehors du discours identitaire, ils risquent de transformer les voix singulières en un Babel narcissique qui ne peut absolument pas concurrencer avec le bruit martial du « Death Metal » patriarcal. 


\section{La pensée féministe}

Là où le discours féministe libéral dit : «C'est injuste, changeons la loi », la pensée féministe radicale demande d'où l'injustice et la violence tirent leur origine. Aussi s'intéresse-t-elle à l'imaginaire, au symbolique, à la philosophie, à la sémantique, au droit, à l'histoire, à la théologie, à la littérature, à l'art, bref à tout ce qui témoigne de l'inscription millénaire de la subjectivité des hommes sur la place publique. Car s'il est important de faire circuler la subjectivité des femmes, il est tout autant impérieux de s'interroger sur ce que cela peut bien être, mondieu, cet homme qui inventa LA femme.

L'objet de réflexion de la pensée féministe c'est la subjectivité masculine et son construit en une raison et une logique qui font l'économie du sujet féminin. Réflexion qui ne va pas sans l'analyse du long récit qui donna lieu à la création de l'incohérent et pourtant incontournable personnage de LA Femme. C'est aussi à la pensée féministe que revient la tâche de comprendre les méthodes et les moyens employés pour reproduire cette raison et cet imaginaire.

Or, penser tout ça n'est pas facile quand le corps tremble de colère et de désarroi, quand la conscience panique devant l'ampleur du mensonge et de l'imposture, quand la mémoire est blessée, quand « le cœur est un coureur solitaire » pour employer un titre de la romancière Carson McCullers.

Or, penser, tout ça n'est pas simple, car la conscience aura à se frayer un chemin entre les messages contradictoires émis par le patriarcat, elle aura à dénouer les mensonges élaborés sur la nature des femmes, à s'interroger sur la crédibilité d'un système de représentation dans lequel les femmes sont décrites sous les traits d'une créature qui serait tout à la fois in/signifiante, fatale, immorale, animale, angélique et monstrueuse.

Tout ça n'est pas clair et il faudra s'attarder à comprendre comment fonctionnent les tactiques d'exclusion/inclusion qui placent les femmes dans la posture inconfortable de la double contrainte, de l'ambivalence, il faudra répertorier les méthodes d'intimidation qui poussent les femmes à l'autocensure, il faudra discerner entre la misogynie, le phallocentrisme et le sexisme ordinaire. Il faudra comprendre la pression exercée par la contrainte à l'hétérosexualité.

Or, penser tout ça n'est pas facile dans une langue qui n'accueille pas volontiers la lucidité désirante du sujet féminin, dans une langue entièrement occupée et habitée par la subjectivité masculine qui s'y pense, s'y amuse, y développe mille et une fantaisies, mille et une contradictions, autant d'utopies, autant de rêves.

\section{La langue}

La langue est un grand réservoir de référents à l'aide desquels on construit son rapport à la réalité, à l'aide desquels on ouvre des portes sur l'imaginaire. Elle est, pour chaque génération, mémoire et virtualité. En principe, la langue appartient à tout le monde. Si, pour une féministe, la langue peut parfois 
ressembler à une assemblée politique « packtée à planche », elle est d'abord pour toutes les femmes un lieu où se modèle (individuellement) et se perpétue (collectivement) notre aliénation. Elle est une école de formation qui prépare au devenir de la colonisée. Car notre langue maternelle et la langue de l'universel ne font qu'une, langue qui exclut et englobe, blesse et rassure, isole et solidarise. Tache aveugle et point de repère.

C'est parce que nous intériorisons la subjectivité des hommes comme une évidence, que nous avons l'obligation de dénouer à l'intérieur de la langue les intrigues qui s'y nouent en notre défaveur. S'il faut du temps pour coloniser, il en faut tout autant pour se sortir de l'aliénation. La blessure est interne et c'est de l'intérieur qu'aura lieu la confrontation, le travail de guérison et de libération. La marge de manœuvre des colonisées que nous sommes est dans la langue même qui nous aliène.

Aussi, je dirais que c'est en se regardant faire avec la langue qu' on parvient à décoder tout ce que je viens d'attribuer à la pensée féministe et que c'est en se regardant être dans la langue qu'on arrive à se faire une image de soi et de notre relation au monde.

Pour cela, il faut indéniablement passer par l'écriture, c'est-à-dire apprendre dans cet acte à puiser de l'énergie là-même où le sujet s'épuise de plaisir et d'angoisse à se faire concurrence dans le vaste monde des images et des idées, et du désir qui les produit.

Il faut passer par l'écriture pour déminer le terrain sémantique, pour examiner soigneusement les mots, pour récupérer, aux sens écologique et médical du terme.

Se regarder faire et se regarder être dans la langue sont des activités qui permettent d'identifier les paradoxes qui immobilisent le sujet féminin. Ce sont des activités qui débouchent sur des questions de présence, de différence, d'identité et de valeurs. Ainsi, celle qui se regarde dans la langue dira :

I. Je sais que je suis/mais je ne suis pas là.

2. Je ne suis pas là/comment apparêtre?

3. Je suis là/mais ce n'est pas moi.

4. Je suis ailleurs. Où ?/Je suis un ailleurs. Lequel ?

Si dans un premier temps, la pensée féministe prend pour objet d'étude le construit de la subjectivité masculine, elle est, dans un deuxième temps, inévitablement amenée à analyser son propre comportement devant les difficultés qu'elle rencontre. Ces difficultés sont nombreuses car le grand principe sur lequel se fonde le discours patriarcal (infériorité des femmes) est protégé par une infinité de petits arguments, de «back up » qui agissent comme des mécanismes parant à toute éventuelle remise en question. Or, c'est en analysant ses réactions et les opérations mentales auxquelles elle doit avoir recours pour résoudre les difficultés qu'elle rencontre, que la pensée féministe se découvre en même temps qu'elle découvre le pot-aux-roses patriarcal. 


\section{Posture}

Tout d'abord, la pensée féministe se découvre morale car elle sait qu'il y a deux choses qu'elle ne peut pas faire pour résoudre ses difficultés : elle ne peut pas inférioriser les hommes et elle ne peut pas faire l'économie du masculin. Certes, elle peut montrer l'irrationalité, la dé/raison du système, faire la preuve de son immoralité mais elle est en quelque sorte condamnée à faire valoir la notion de différence et à reconnaître l' Autre.

Or, au fil de son analyse, la pensée féministe doit inévitablement sonder le féminin sans le détacher de la réalité des femmes et, ce faisant, elle se découvre absolument passionnée par son sujet. Aussi lui arrive-t-il de dériver, de se laisser aller à imaginer des scénarios dans lesquels les femmes ont tous leurs esprits, toute leur énergie, sont de toute beauté, libres comme l'air.

En s'éloignant de la tâche qu'elle s'était initialement donnée, la pensée féministe se met à déborder d'émotion et de vie, à jouer avec les mots, à produire des métaphores inusitées, à élaborer des hypothèses festives, à concevoir du sens là où, en principe, il ne devrait pas y en avoir. Se faisant, elle est amenée à chercher un langage propice à rendre compte de ce genre de débordement. Aussi, dérive-t-elle, à l'occasion, dans cet espace où tout est toujours virtuel : la fiction. Là où la pensée féministe pensait uniquement avoir affaire à l'Homme, voici qu'elle se passionne pour les femmes comme sujet d'intérêt, de reconnaissance et de désir. Ce débordement est indéniablement lesbien. Il est une dérive majeure qui permet à la pensée féministe de re/doubler de motivation, d'ardeur, d'adresse et de créativité.

On pourrait alors dire que de par son va-et-vient entre la subjectivité masculine et la subjectivité féminine, de par ses hésitations devant la « nature » des hommes et celle des femmes, de par son insistance à faire valoir une différence qui n'engendre pas le rejet de l'Autre, la pensée féministe offre, en théorie, un débouché à la pensée masculine.

Ainsi que dans le cas de la subjectivité des femmes qui, comme nous l'avons vu, est amenée à servir de pont entre hommes, les modalités de travail de la pensée féministe peuvent inspirer quelques métaphores au philosophe contemporain.

Mais ne nous méprenons pas : ce qui sera investi par le penseur ce n'est pas la pensée féministe, non plus que le féminin, mais la posture féminine révélée par la pensée féministe. La posture féminine sera investie par le masculin parce qu'elle est on ne peut plus appropriée pour traduire, en cette fin de siècle, l'inconfortable réalité du sujet masculin qui est encore, mais qui n'est plus là comme avant, qui est là maintenant comme un j'autre. En empruntant une posture féminine, le philosophe contemporain pourra traduire le vertige de la perte, pourra s'inventer une identité de minoritaire, cultiver une marginalité, faire valoir sa différence et réaffirmer son intégrité dans un monde éclaté, kaléidoscopique. Aussi, le philosophe contemporain peut-il, tout en se mettant à la place de l'Autre (cette fois-ci selon la formule bien féminine de la 
compassion), réapparêtre comme un dissident dans le champ de la raison ancienne (pour les femmes il sera alors perçu comme un alliè) et renaitre dans un ailleurs qu'il aura tôt fait de meubler de sa subjectivité masculine nouvellement « liftée ».

Je ne dis pas qu'il y a là préméditation ou complot de la part du philosophe, je dis tout simplement que la pensée féministe ne peut pas compter sur le philosophe et qu'elle fait mieux de compter sur son propre débordement. Car, à être depuis tout ce temps dans la marge, la pensée féministe ne peut advenir ailleurs qu'en se tenant au bord, à l'extrême limite du sens ou en le faisant constamment déborder. Bien que cela puisse sembler paradoxal, c'est le débordement lesbien qui donne à la pensée féministe ses racines aériennes, son élan et son focus. Les textes majeurs du féminisme émanent de ce débordement comme si l'utopie était la seule forme affirmative du féminin, capable de déplacer le sujet féminin de manière à ce que, dit ou non-dit, il soit omniprésent comme une source de sens virtuel, une ouverture incontournable vers l'ailleurs.

\section{Le discours féministe}

Le discours féministe s'inspire du vécu des femmes et de leur témoignage et il se nourrit de la pensée féministe. Là où les femmes expriment leur désarroi, là où la pensée féministe montre les rouages du patriarcat, le discours féministe tire des conclusions et passe à l'action. Là oủ les femmes blâment un mari, un amant, un père ou un employeur, là où la pensée féministe incrimine le système patriarcal, le discours féministe accuse les hommes. Là où la pensée féministe découvre les lois qui perpétuent l'aliénation des femmes, là où les femmes révèlent le drame de la condition féminine, le discours féministe dénonce les institutions qui contribuent à la domination et à l'exploitation des femmes. Il analyse leurs effets, propose des changements. Le discours féministe « objectivise » la subjectivité des femmes et il politise les assertions de la pensée féministe. Il ose porter des jugements. Il s'arroge le droit de généraliser. Il prend en charge la colère, la révolte, les larmes, les espoirs, l'humour et les utopies des femmes. Il « crie des noms ». Il polarise. Il exacerbe les différences, il divise, il crée des solidarités. Il est tout à la fois normatif, iconoclaste, provocateur et responsable. Il change les règles du jeu, les rôles, la représentation. Il fait la fête. Il fait la morale. Il est multiple.

On comprend donc pourquoi on l'accuse d'être irrationnel, pourquoi il dérange tant. Pourquoi il est souvent l'objet d'une méfiance de la part des femmes et en quoi il fait toujours l'objet d'une énorme résistance, sinon d'une opposition hystérique de la part des hommes.

Le discours féministe entretient une tension sociale et psychique en plaçant en évidence et à la vue de tous ce qui divise économiquement, ce qui sépare culturellement, ce qui désunit les hommes et les femmes. Il est étonnant qu'on accuse le féminisme de contribuer à la discorde dés hommes et des femmes alors qu'il s'applique, en montrant ce qui les divise, à redonner aux 
femmes leur humanité. En fait, le discours féministe entretient sur la place publique un débat sans lequel (et cela l'histoire l'a démontré) l'assujettissement des femmes redevient monnaie courante.

Ce qu'on ne pardonne pas au discours féministe, c'est d'avoir politisé les rapports les plus intimes et les plus quotidiens. C'est d'avoir introduit la notion de pouvoir dans des rapports antérieurement considérés comme découlant d'un ordre « naturel ». C'est de s'être attaqué à un consensus idéologique et à un ordre social en passant directement par l'individu, c'est-à-dire en demandantà ce dernier non seulement de changer ses habitudes mais aussi sa façon d'imaginer les rapports sexués. Aussi le discours féministe est-il perçu comme moralisateur, comme une intrusion au cœur de ce qu'il y a de plus intime chez tous.

Mais à l'encontre de la majorité des discours politiques, qui visent en définitive la prise réelle d'un pouvoir politique, le discours féministe cherche à redistribuer le pouvoir entre les hommes et les femmes. Pour cela, il doit inévitablement s'en prendre au pouvoir machiste et phallocentrique. Pour redonner aux femmes un pouvoir sur leur vie et sur leur corps, il doit interroger toutes les formes d'activités où le pouvoir mâle s'est installé d'office. Aussi le discours féministe remettra-t-il en question la sainte institution du mariage là où s'entassent pêle-mêle l'amour, le travail domestique, la sexualité, l'économie, l'affectivité, la maternité, le soin et l'éducation des enfants. Évidemment, ce pouvoir des femmes sur elles-mêmes ne peut être récupéré sans contrarier directement ceux qui croient le détenir d'autorité divine, naturelle ou légale et j'irais même jusqu'à dire sans contrarier celles qui vivent dans la certitude qu'elles possèdent déjà ce pouvoir.

Le discours féministe va directement au cœur des femmes et des hommes. Il touche aux fibres les plus sensibles de nos émotions, de notre affectivité, il touche l'image que chacune et chacun se fait de soi à travers une appartenance à un genre.

Or, le discours féministe est partisan, c'est-à-dire qu'en plus de prendre parti pour les femmes contre les hommes violents, violeurs et dominateurs, il interpelle et accuse virtuellement les hommes qui refuseraient de remettre en question un pouvoir et des privilèges illégitimement acquis. Il provoque ainsi chez les hommes une gamme de sentiments troubles qui oscillent entre la compréhension, la culpabilité, la colère ou un sentiment de perte d'identité.

Parce qu'il est partisan le discours féministe exacerbe les différences, il divise, il crée des solidarités.

En s'appuyant sur les témoignages des femmes et sur les premiers constats de la pensée féministe, le discours féministe en est arrivé dans un premier temps à montrer les différences, puis il les a exacerbées en revalorisant le féminin et ce qui s'y rattache ainsi qu'en discréditant le masculin et ce qui y est généralement associé. Cette formule relativement simpliste (je fais ici remarquer que bien que pratiquée de tout temps par les groupes dominants, cette formule se voit uniquement condamnée lorsque utilisée par les groupes 
dominés), cette formule donc a quand même l'avantage de tisser des liens nouveaux entre les membres du groupe dominé et ainsi de créer une solidarité face à un « ennemi » commun, solidarité qui engendre énergie et projets qui permettent de passer à l'action.

Or, une fois la subjectivité des femmes objectivée par le discours féministe autour d'un projet commun : lutter contre le sexisme, contre le patriarcat, contre la misogynie, les femmes n'ont pas cessé pour autant de s'interroger sur les conditions objectives de leur vie, sur leurs émotions et sur les rapports de pouvoir. A nouveau, les femmes vont recourir à l'expression de leur vécu pour rendre compte de besoins spécifiques et témoigner de la position qu'elles occupent dans la hiérarchie sociale.

Ainsi vont-elles nommer les disparités et les différences qui existent entre elles, différences qui, cette fois-ci, découlent de leur appartenance à un groupe social opprimé et discriminé. Certaines de ces différences seront débattues idéologiquement à travers les filtres conçus au masculin du marxisme, de la psychanalyse et du nationalisme mais d'autres - ce sont les plus importantes, car elles mettent en cause le corps, la sexualité, l'image de soi-le seront viscéralement. C'est ainsi que les féministes lesbiennes et les féministes afroaméricaines, en remettant en question le discours des féministes blanches et hétérosexuelles de la petite bourgeoisie, exacerberont une autre série de différences, créeront de nouvelles solidarités, diversifieront le discours féministe et son champ d'action et nourriront de nouvelles tensions, qui, aux États-Unis et au Canada, trouveront partiellement leur résolution dans la création du concept « politically correct ».

On se moque beaucoup par les temps qui courent de ce concept récemment apparu dans la configuration politique du Québec français. On s'amuse à le confondre avec une forme de morale égalitariste qui nivellerait désirs, imagination, fantaisies, qui interdirait tout débordement, tout écart. Pourtant, le concept « politically correct » vise essentiellement à accroître la participation et le développement des individus appartenant à des groupes infériorisés et marginalisés. C'est un concept qui permet le développement de discours minoritaires et marginaux mais qui permet aussi de relire l'histoire selon des angles différents. Il engendre une nouvelle dynamique dans les rapports de force et nous ne connaissons pas encore les effets de cette dynamique : Babel, métissage ou nouvelle territorialité identitielle ? Là encore, nous ne savons pas ce qu'il restera pour les femmes de ce nouveau concept développé entre féministes.

Le travail féministe servira-t-il encore une fois de pont entre hommes blancs, noirs, autochtones et des minorités ethniques? En principe, nous aurions raison de nous réjouir d'une telle influence, mais pourra-t-on vraiment parler de changement culturel si ne se rencontrent que des cultures misogynes et sexistes? Pas facile n'est-ce pas de sortir de la marginalité?

Le problème est de taille : chaque fois que le féminisme réussit à disséminer dans la société des propositions fondées sur le respect de l'Autre 
et de la différence, ces propositions, d'abord initiées en fonction de la différence sexuelle, se transforment peu à peu en affaires courantes dont on évacuera à moyen terme le féminin.

Si on peut affirmer que chaque fois que le système patriarcal intègre des valeurs féminines, toutes et tous en profitent, on peut cependant se demander si le féminisme est irrémédiablement condamné à ouvrir des portes sur le champ des possibilités relationnelles, c'est-à-dire à jouer un rôle de médiation. Ainsi, on pourrait dire que, dominées, les femmes servent de monnaie d'échange entre hommes; en possession de leurs esprits, elles ne peuvent aspirer qu'à être des hautes-fonctionnaires compétentes et influentes mais sans pouvoir de décision, ce qui n'est pas sans rappeler la position traditionnelle de la femme forte, celle, dit-on, qui se cache toujours derrière un grand homme.

Politiquement, on pourrait ainsi affirmer que : au milieu des hommes, les femmes sont invisibles; au côté des hommes, elles sont marginalisées; séparatistes (au sens lesbien du terme), elles sont ignorées; opposées aux politiques sexistes, c'est-à-dire féministes, elles sont menacées de représailles. On comprend donc pourquoi le discours féministe est tout à la fois normatif, iconoclaste, provocateur et responsable. Parce que le discours féministe est alimenté par la question de l'identité, laquelle interroge constamment la référence, la différence et l'environnement, ce discours demeure un espace ouvert. Je dirai aussi que comme tout discours qui procède d'une interrogation sur la réalité et l'imaginaire, le discours féministe a aussi besoin de poésie. Il n'y a rien d'immoral à ce que le discours féministe soit parfois plus surréaliste qu'un poème de Breton, plus baroque qu'une sculpture du Bernin, plus répétitif que la musique de Steve Reich, plus mouvementé qu'une chorégraphie de La La La Human Steps, plus rapaillé qu'un poème de Gaston Miron. L'émotion, j'y tiens.

\section{«Backlash 》}

Qui dit «backlash », dit remise en question d'un mouvement progressiste, dit retour à une convention antérieure. Reconnaître qu'il existe présentement un «backlash » contre le féminisme, c'est admettre que le féminisme est bien vivant ou qu'il a déjà trop influencé la société. Mais à quoi évalue-t-on un «backlash »? A quelques articles anti-féministes, à un retour en force d'images et de propos misogynes? Aux coupures budgétaires subies par les groupes de pression et d'action? A des législations qui remettent en question les droits acquis des femmes?

Avant de répondre à ces questions, il me semble important de rappeler que la société québécoise moderne a zo ans et que le féminisme radical en a 24. Concrètement, cela veut dire qu'ils ont plus (au masculin) ou moins (au féminin) grandi ensemble. Cela signifie que les femmes et les hommes québécois sont allés à l'université ensemble, ont cessé d'aller à l'église en même temps; qu'ils ont connu la libération sexuelle et la contraception en même temps; qu'ils ont été amenés à s'interroger sur l'identité, à parler de domination, d'exploitation et de colonisation, qu'ils ont plus ou moins vécu ensemble leur 
crise marxiste, ont connu en même temps un engouement pour la psychanalyse, que les deux (l'homme qui rêvait d'un pays qui ait du bon sens et la femme qui rêve d'une vie qui a du bon sens) ont contribué à renouveler et à enrichir la littérature québécoise. Aujourd'hui, tous les deux s'étonnent et s'inquiètent de l'individualisme et de l'érosion des solidarités. En somme, l'homme québécois n'a jamais eu une grande longueur d'avance sur les femmes. Bien sûr, il est toujours premier au fil d'arrivée avec, si vous me permettez l'expression, une verge d'avance. C'est cette petite avance qu'il ne veut pas perdre et qui le rend nerveux, méfiant, encore violent. Cette petite avance, elle peut s'accroître rapidement dans le contexte d'un nationalisme qui tend de plus en plus à devenir défensif. Les nationalismes, c'est bien connu, n'ont que faire des femmes. Il ne faut pas confondre la période libérale et libertaire des années ig6o avec le nationalisme. C'est la quête d'identité particulièrement exprimée par les poètes qui a permis l'éclatement des valeurs traditionnelles et de transformer le discours nationaliste en un espace ouvert. Et comme c'est presque toujours le cas, le féminisme a pu prendre racine dans la société québécoise grâce à cet éclatement des valeurs. La même chose s'est passée aux États-Unis avec le Civil Rights Movement et en France avec Mai 68. Parallèlement au féminisme américain qui s'est développé idéologiquement autour du pragmatisme et du lesbianisme, du féminisme français qui s'est pensé en fonction du marxisme et de la psychanalyse, le féminisme québécois s'est, dans un premier temps, articulé en combinant le discours de l'identité et celui du marxisme. Puis, il a emprunté aux discours américain et français pour ensuite s'exprimer dans le champ de la création littéraire. Ce que nous appelons l'écriture au féminin est un phénomène québécois qui a permis à la conscience. féministe de s'exprimer d'emblée dans le champ de la modernité, d'interroger la langue et de faire advenir en celle-ci de nouvelles propositions formelles et thématiques. En d'autres termes, le féminisme a pénétré la culture québécoise par l'intermédiaire de subjectivités singulières exprimant une conscience féministe. Ce qui, dans un sens, lui a permis d'être relativement crédible au cœur de la turbulence idéologique qui balayait alors le Québec.

En dehors de la littérature, le féminisme a aussi été associé à des figures singulières de femmes fortes dont les propos féministes, étaient pour la plupart rattachés à une autre cause, nationaliste ou syndicaliste.

Bien que les lesbiennes aient toujours été présentes et très actives dans le mouvement, le féminisme québécois a toujours eu un visage hétérosexuel. Exception faite de quelques auteures, de la revue Lesbiennes d'hier et Amazones d'aujourd'hui et de quelques articles, le féminisme québécois n'est jamais « allé trop loin ». Il est resté « crédible » et a pu devenir ainsi un interlocuteur valable. Certes, il a rencontré de l'opposition, mais il a bénéficié de l'élan libertaire donné par la Révolution tranquille. Comme le fait remarquer Diane Lamoureux dans son livre Fragments et collages, il a eu très tôt partie liée avec les instances gouvernementales afin d'offrir aux femmes des services urgents. 
Dans un sens, on peut dire que le féminisme québécois a tenu parole à deux niveaux : pragmatique et littéraire.

Là où le féminisme québécois est pour ainsi dire défaillant, c'est au niveau théorique et critique. Les féministes radicales, persuadées du bienfondé de leurs convictions, n'ont pas senti le besoin de pousser leur analyse et s'en sont remises aux écrits américains pour leur évolution personnelle alors que les féministes égalitaires (majoritairement de la classe moyenne) n'ont pas vu la nécessité de dépasser la mesure et de risquer ainsi de perdre leur crédibilité. Le résultat est une pénurie de textes théoriques et critiques, pénurie qui aura pour effet de créer un vide entre les générations. Je peux me tromper, mais il me semble que les jeunes étudiantes anglophones de Montréal qui continuent d'être alimentées en textes féministes américains débattent plus facilement en termes féministes la réalité sociale. Dois-je, quant à notre défaillance théorique, faire intervenir ici le difficile rapport que les Québécois et les Québécoises, en général, ont à la langue française écrite ? Je ne sais. Mais une chose est certaine : le Québec produit peu de discours théoriques et critiques. Qu'il n'y ait aucune librairie féministe à Montréal, aucun journal ou magazine de théorie féministe, que les cours d'Études féminines ou féministes se comptent sur les doigts de la main, cela traduit un terrible manque de perspective et de continuité. Aussi, lorsque le discours féministe réapparaît dans sa dimension radicale, comme ce fut le cas après le massacre de Polytechnique, il donne l'impression d'être parlé par des dinosaures ou des martiennes. Je pense que nous allons payer très cher notre silence des dernières années, que nous devons à tout le moins nous interroger sur la façon que nous avons de relaxer devant ce que nous croyons être des acquis permanents, de déléguer notre droit de parole à des lobbyistes ou de croire que nous sommes allées trop loin. Car même dans l'hypothèse bien fictive que nous soyons enfin en pleine possession de tous nos moyens (affectifs, intellectuels, économiques), rien ne justifie notre retrait de la place publique en tant que féministes. C'est ce retrait du lieu public qui produit ce que j'appellerais le syndrome du fait divers, c'està-dire, qui permet graduellement de réinsérer les histoires de femmes sous la rubrique fait divers. Le syndrome du fait divers est une façon de dépolitiser les conditions de vie des femmes.

Plus j'y pense, plus je me dis que si, en effet, nous sommes de plus en plus en possession de tous nos moyens, c'est qu'alors nous n'avons plus aucune excuse pour ne pas intervenir directement dans la conduite des affaires de l'Etat tout en continuant d'être responsables devant les affaires courantes de la vie. Or, ceci ne peut pas se faire sans un environnement féministe. Sans cet environnement, chaque femme, forte et fière, s'épuise rapidement. Il faut de la pensée, du discours et l'énergie de la fête et du plaisir, pas seulement des rapports officiels et des statistiques.

Aujourd'hui, nous nous étonnons d'un «backlash » comme nous avons été incrédules devant le massacre de Polytechnique. Pourtant, les hommes du «backlash » qui sont (comme le fait remarquer Marisa Zavalloni dans un de ses 
textes) dans l'ensemble des hommes instruits, en principe non violents euxmêmes, pourtant ces hommes ne disent rien de nouveau. Ils réaffirment, cette fois-ci avec des airs de victimes, que les femmes doivent rester à leur place car pour être valorisé un homme doit se sentir important, entendre ici plus important. Ils rappellent dans un même souffle que l'agressivité masculine est une forme universelle de transcendance ou une nécessité biologique. Ils disent que lorsque les femmes s'épanouissent et commencent à avoir un peu de plaisir dans la vie, cela déséquilibre les hommes. Bref, la morale de l'histoire c'est que la morale du bonheur est dans la subordination des femmes aux hommes. Ce que les hommes du «backlash » ont le mérite de nous rappeler, c'est que leur besoin de contrôler est fondamentalement non négociable. Ce que les femmes qui acquiescent aux propos des hommes du «backlash » ont le mérite de nous rappeler, c'est à quel point il est difficile de faire comprendre que les enjeux du féminisme sont infiniment plus grands qu'un salaire égal et n'ont rien à voir avec le fait de s'être trouvé un « chum le fun » ou un bon mari.

\section{De l'enthousiasme, du débordement : l'espoir, le futur}

Dans son article «Praxis de la différence » (Les Cahiers du GRIF n 46, printemps 1992), Françoise Collin affirmait : « la pire condamnation de l'opprimé, de quelque oppression qu'il relève, est sa condamnation au politique ». Ce qui veut sans doute dire être condamnée à jouer des coudes alors qu'on aimerait tout simplement jouer du cœur et de l'esprit, ce qui signifie ne pas avoir le loisir de perdre de vue les rapports de force. Soit, j'accepte d'être condamnée au politique, mais j'accepte surtout d'être condamnée à la créativité dans un espace imaginaire qui contraint le mouvement des pensées du sujet féminin. Jaccepte d'être condamnée à chercher des solutions heureuses côté futur, côté splendeur de vivre. J'accepte de parler très simplement du bonheur. Naturellement, j'accepte de parler de l'angoisse.

Dans un monde éclaté où nos points de repère historiques, idéologiques et symboliques ne sont plus que des empreintes visuelles assujetties au mouvement de nos paupières dont le battement est réglé par les tilts et les flashes en accéléré de la post-modernité, il faut bien se dire que si la réflexion féministe a trouvé quelques échos à ses propositions, nous n'avons pas encore trouvé de solution à la marginalisation du féminin; et que plus le temps passe, plus nos questions et nos réponses risquent d'être ponctuelles, superficielles. Car, nous aussi sommes entrées dans un décalage temporel qui nous laisse là, bouche ouverte d'étonnement entre la lenteur de nos réflexes et l'accélération des stimuli et des questions sans réponse qui surgissent, paradoxalement, futiles et urgentes.

Du futur, je ne sais qu'une chose c'est qu'il faudra apprendre à ralentir, prendre le temps de prendre son temps pour interroger la science, la politique, nos besoins réels. Prendre le temps de laisser monter la pensée et le silence comme jadis on laissait monter le désir. 
J'ai souvent employé le mot « espoir » dans mes textes presque d'une manière incantatoire pour m'assurer que nos pensées et nos gestes aient un futur. Pourtant, je suis une femme du présent qui cherche à comprendre ce qui confine chaque génération de femmes dans des thèmes, des métaphores, des comportements qui répètent notre aliénation, malgré tout notre courage. J'imagine la passion du langage qui permet d'en sortir. Le tourment qui ouvre des brèches dans l'histoire. Le délire qui consume les lieux communs. J'imagine l'urgence intérieure qui oblige à liquider les truismes patriarcaux. La pensée est le fruit d'un déplacement de l'appartenance dans une appartenance qui invente son horizon. Je me déplace toujours à partir des mots de mon appartenance. Je tiens pour acquis que je dois faire avec la langue dans l'état où elle est. Je tiens pour acquis que la langue devra faire avec ce quej'inventerai en elle.

Je suis une femme du présent car le plaisir que j'ai de vivre au présent s'accompagne toujours de l'idée que la vie est, dans sa complexité même, une garantie de renouveau. J'imagine alors que penser, imaginer et s'émouvoir constituent une forme de contribution à ce rêve récurrent qui permet d'entretenir l'espoir que nous pouvons changer le cours de l'imaginaire. Car il me semble que ce qui donne à l'espèce humaine sa complexité et qui la rend toujours virtuelle, c'est sa faculté d'imaginer, c'est le nombre incalculable de permutations d'images et de mots dont nous sommes capables pour donner un sens à la vie. Je refuse d'imaginer le pire, c'est-à-dire que nous soyons inaptes à imaginer le futur de l'espèce autrement qu'au masculin universel. Je réclame le courage et la sagesse patiente de nos ancêtres-mères. Je réclame l'intelligence, l'imagination, la créativité et la lucidité de mes contemporaines.

Parce que la féministe que je suis a toujours partie liée avec la poésie, elle continue de rêver, de tisser des liens entre la réalité et la fiction, d'ouvrir des brèches au cœur même de ces liens de manière à ce qu'ils puissent, mobiles, mobiliser le désir, l'ouvrir sur l'horizon. Écrire je suis une femme, est plein de conséquences. 